\title{
DERMATÓGLIFOS EM CRIANÇAS COM CONSTIPAÇÃO CRÔNICA
}

\author{
Soraya GOSHIMA, Ulysses FAGUNDES-NETO e Mauro Batista de MORAIS
}

\begin{abstract}
RESUMO - Racional - Os dermatóglifos podem ser utilizados na avaliação da transmissão genética de determinadas doenças, sendo controversa na literatura a associação entre o padrão arco e constipação. Objetivo - Comparar os padrões de dematóglifos de crianças com constipação crônica com os de crianças sem constipação e com o hábito intestinal e padrões de dermatóglifos de suas mães. Métodos - Foram estudados três g rupos de crianças com idade entre 2 e 12 anos e suas respectivas mães: 35 pacientes com constipação grave, 45 crianças com constipação leve e 51 crianças com hábito intestinal normal, que constituíram o grupo-controle. Preencheu-se ficha padronizada com informações gerais e do hábito intestinal das crianças e de suas mães. Obtiveram-se as impressões digitais das crianças e de suas mães, que foram classificadas em arco, presilha radial, presilha ulnar, ver ticilo e outros.Resultados - A freqüência do padrão arco foi semelhante nos três grupos estudados: constipação g rave (25,7\%), constipação leve $(28,9 \%)$ e controles (23,5\%). Hábito intestinal compatível com constipação foi observado em 51,9\% (68/131) das mães. Arco foi observado em 35,3\% das mães com constipação e em 42,9\% das com hábito intestinal normal. Foi observada concordância muito le ve entre a ocorrência de constipação na criança e na mãe. Conclusãa - Não se observou associação entre a presença de arco e constipação, tanto nas crianças como em suas mães, sendo muito leve a concordância entre a ocorrência de constipação na criança e em suas mães biológicas.
\end{abstract}

DESCRITORES - Dermatóglifos. Constipação, genética. Criança.

\section{INTRODUÇÃOO}

A fisiopatologia da constipação crônica funcional na infância é complexa e de etiologia multifatorial, acreditando-se que resulte da interação de fatores dietéticos, ciclo vicioso de evacuação dolorosa e comportamento de retenção fecal, distúrbio da motilidade intestinal e características constitucionais e genéticas $^{(2,11,15)}$. Há poucos estudos que abordam a participação de fatores genéticos na constipação crônica funcional. $\mathrm{O}$ início da constipação nos primeiros meses de vida ${ }^{(16)}$, observado por vários autores ${ }^{(12,13,14)}$, é considerado um indício da influência das características constitucionais e genéticas na gênese da constipação. Além disso, estudo realizado em gêmeos mostrou que os monozigóticos apresentam concordância de constipação 4 vezes maior do que os dizigóticos ${ }^{(3)}$.

Dermatóglifos ou impressões digitais são conf igurações lineares finas encontradas nas polpas digitais e nas regiões palmar e plantar ${ }^{(1)}$. A área mais estudada são as polpas digitais e os principais padrões de dermatóglifos são arco, verticilo, presilha ulnar e radial ${ }^{(1)}$. Os dermatóglifos podem ser utilizados para auxiliar no estabelecimento do papel da transmissão genética em várias doenças familiares como em patologias cardíacas $^{(20)}$, síndrome de Down ${ }^{(18)}$, doença celíaca ${ }^{(6,21)}$, pseudo-obstrução intestinal familiar crônica ${ }^{(5)}$ e úlcera duodenal $^{(9)}$. Por sua vez, existem três estudos publicados avaliando a relação entre constipação e padrões de dermatóglifos ${ }^{(7,8,19)}$. No primeiro estudo ${ }^{(8)}$, constatou-se maior frequiência do padrão arco em adultos com constipação com início anterior aos 10 anos de idade e dor abdominal. Outro estudo $^{(19)}$, realizado com crianças com constipação e dor abdominal, confimou a associação com o dermatóglifo do tipo arco. No terceiro ${ }^{(7)}$, envolvendo crianças, não se confirmou a associação entre constipação e maior freqüência de arco, caracterizando uma situação controversa na literatura.

Levando em consideração as informações acima mencionadas, foi elaborado este estudo com os objetivos de: 1. comparar os padrões de dermatóglifos de crianças com constipação crônica com os de crianças sem constipação, e 2. estudar a relação entre a ocorrência de constipação crônica e do dermatóglifo do tipo arco destas crianças com suas mães biológicas.

Disciplina de Gastroenterologia Pediátrica do Departamento de Pediatria da Universidade Federal de São Paulo - Escola Paulista de Medicina, São Paulo, SP. Endereço para correspondência: Dra. Soraya Goshima - Rua Baltazar da Veiga, 609 - ap. 92 - Vila Nova Conceição - 04510-001 - São Paulo, SP, Brasil 


\section{CASUÍSTICA E MÉTODOS}

Este é um estudo transversal no qual se analisaram os padrões de der matóglifos de 131 crianças com idade entre 2 e 12 anos distribuídas em três grupos:

1. Grupo com constipação g rave - constipação crônica funcional em atendimento no ambulatório de constipação da Disciplina de Gastroenterologia Pediátrica da Universidade Federal de São Paulo - Escola Paulista de Medicina (UNIFESP-EPM), constituído por 25 meninos e 10 meninas;

2. Grupo com constipação leve - constipação crônica, identificada por rastreamento ativo em serviço de pronto atendimento pediátrico durante consulta em que a queixa não se relacionava com distúrbios do hábito intestinal, constituído por 23 meninos e 22 meninas;

3. Grupo-controle - com hábito intestinal normal, ou seja, sem constipação ou diaréia, que realizaram consulta no pronto atendimento pediátrico com queixas não relacionadas ao sistema digestório, constituído por 22 meninos e 29 meninas.

Analisaram-se, também, os padrões de dermatóglifos e o hábito intestinal das mães biológicas de todas as crianças incluídas no estudo.

Constipação crônica na criança foi caracterizada pela eliminação de fezes endurecidas, acompanhada de dor, medo ou dificuldade durante as evacuações, associadas ou não com escape fecal, sangramento em torno das fezes e intervalo entre as evacuações maior ou igual a 3 dias, por período maior do que 3 meses $^{(14)}$.

Foram considerados os seguintes critérios de exclusão: crianças não acompanhadas pela mãe biológica ou por tadoras de encefalopatia crônica, hipotiroidismo, diabetes mellitus, hipercalcemia, hipocalemia e constipação orgânica de causa intestinal e, ainda, que estivesse utilizando medicamentos que pudessem provocar constipação.

Para todas as crianças, realizou-se um inquérito individual com informações gerais e do hábito intestinal, onde constavam questões sobre a consistência, formato e calibre das fezes, freqüência e características das evacuações.

Foram coletadas também informações sobre o hábito intestinal da mãe, que foi considerada portadora de constipação, quando, em geral, apresentava evacuações de fezes endurecidas com dor ou dificuldade e/ou número de evacuações menor ou igual a três evacuações por semana ${ }^{(22)}$.

A coleta das impressões digitais das crianças e das mães foi realizada segundo as recomendações clássicas ${ }^{(23)}$ e analisadas por técnicos especializados do Departamento de Datiloscopia do Serviço de Identificação da Polícia Federal (São Paulo) e classificadas em arco, presilha radial, presilha ulnar, verticilo e outros que incluíam anômalo, amputação e cicatriz.

A análise estatística foi realizada com o empre go dos programas de computador EPI-INFO versão 6.0 e Jandel Sigma-Stat ${ }^{\circledR}$. Os testes utilizados são mencionados em cada um dos resultados. A intensidade de concordância de constipação e do dermatóglifo do tipo arco nas crianças e respectivas mães, foi avaliada com o emprego do coeficiente Kappa, considerando os seguintes graus: $+0,0$ a +0,2, concordância muito leve; $+0,21$ a $+0,40$, leve; de $+0,41$ a $+0,60$, moderada; de $+0,61$ a $+0,80$, intensa e de $+0,81$ a $+1,00$, concordância quase perfeita ${ }^{(10)}$.

\section{RESULTADOS}

Não se observou diferença estatisticamente significativa na média da idade dos g rupos com constipação g rave, com constipação leve e do grupo-controle que foram, respectivamente, 71,2 $\pm 38,0,73,3 \pm$ 33,6 e 74,0 $\pm 34,0$ meses ( $P>0,05$, teste de Kruskal-Wallis). Quanto ao sexo, de acordo com a partição do Qui-quadrado, a proporção de pacientes do se xo masculino no grupo com constipação g rave $(71,4 \%)$ foi maior $(P<0,05)$ do que nos grupos com constipação leve $(51,1 \%)$ e controle $(43,1 \%)$. A utilização atual e prévia de laxantes foi maior nos pacientes com constipação g rave $(37,1 \%$ e $74,3 \%$, respectivamente), do que nas crianças com constipação leve (2,2\% e 33,3\%), sendo ambas as diferenças estatisticamente significativas segundo o teste do Qui-quadrado $(P<0,001)$. Quanto à média da frequiência de evacuações nos grupos com constipação grave, leve e controle observou-se, respectivamente, $3,4 \pm 3,3,4,8 \pm 2,0$ e $6,4 \pm 1,8$ evacuações por semana ( $P<0,001$, teste de Kruskal-Wallis). O teste de comparações múltiplas mostrou que a frequiência de evacuações das crianças com constipação g rave é estatisticamente menor do que a das com constipação le ve que, por sua vez, é menor do que a do grupo-controle. Em relação à percentagem de pacientes que iniciaram o quadro de constipação antes dos 12 meses de idade, foram encontrados $45,7 \%$ no gr upo com constipação grave e 40,0\% no g rupo com constipação leve ( $P=0,78$, teste do Qui-quadrado)

A ocorrência dos diferentes padrões de dermatóglifos das crianças dos três g rupos está apresentada na Tabela 1. A ocorrência de arcos nos três grupos não apresentou diferença estatisticamente significativa. A análise do número de arcos em todos os dígitos nos três grupos também não diferiu de maneira estatisticamente signif icativa: constipação grave: $25(7,1 \%)$ arcos em 350 dígitos, constipação leve: 31 (6,9\%) em 450 dígitos e grupocontrole: $35(6,9 \%)$ em 510 dígitos.

Constipação foi caracterizada em 68 (51,9\%) das 131 mães. A ocorrência dos diferentes padrões de dermatóglifos nas mães das crianças dos três grupos de crianças, segundo o hábito intestinal da mãe, está apresentada na Tabela 2, não se observando associação entre constipação na mãe e presença de arcos. Quanto ao número total de arcos nos 10 dígitos das mães, observ aram-se 63 (9,3\%) arcos nos 680 dígitos de mães com e em 77 (12,2\%) dos 630 dígitos das mães sem constipação. 
TABELA 1 - Presença dos diferentes padrões de dermatóglifos nas crianças dos g rupos com constipação grave, leve e controles

\begin{tabular}{lccc}
\hline $\begin{array}{l}\text { Padrão presente em } \\
\text { pelo menos 1 dos 10 dígitos }\end{array}$ & $\begin{array}{c}\text { Constipação grave } \\
(\mathbf{n}=\mathbf{3 5})\end{array}$ & $\begin{array}{c}\text { Constipação leve } \\
(\mathbf{n = 4 5})\end{array}$ & $\begin{array}{c}\text { Grupo-controle } \\
(\mathbf{n = 5 1 )}\end{array}$ \\
\hline Arcos & $9(25,7 \%)$ & $13(28,9 \%)$ & $12(23,5 \%)$ \\
Presilha radial & $9(25,7 \%)$ & $9(20,0 \%)$ & $11(21,6 \%)$ \\
Presilha ulnar & $35(100,0 \%)$ & $44(97,8 \%)$ & $47(92,2 \%)$ \\
Verticilo & $28(80,0 \%)$ & $36(80,0 \%)$ & $37(72,5 \%)$ \\
Outros & $1(2,9 \%)$ & $0(0,0 \%)$ & $2(3,9 \%)$ \\
\hline
\end{tabular}

Proporção de arcos: constipação grave (9/35) versus constipação leve (13/45) ver sus grupo-controle (12/51); teste do Qui-quadrado: $P=0,836$

TABELA 2- Presença dos diferentes padrões de der matóglifos nas impressões digitais das mães de acordo com a presença ou não de constipação

\begin{tabular}{lcc}
\hline \multirow{2}{*}{$\begin{array}{l}\text { Padrão presente em } \\
\text { pelo menos } 1 \text { dos 10 dígitos }\end{array}$} & Constipação \\
\cline { 2 - 3 } & Sim (n= 68) & Não (n= 63) \\
\hline Arco & $24(35,3 \%)$ & $27(42,9 \%)$ \\
Presilha radial & $16(23,5 \%)$ & $9(14,3 \%)$ \\
Presilha ulnar & $66(97,1 \%)$ & $60(95,2 \%)$ \\
Verticilo & $45(66,2 \%)$ & $45(73,0 \%)$ \\
Outros $\geq 1$ & $1(1,5 \%)$ & $4(6,3 \%)$ \\
\hline
\end{tabular}

Proporção de arcos: constipação (24/68) versus sem constipação (27/63); teste do Qui-quadrado: $P=0,479$

Para a análise da concordância da presença de constipação nas crianças e mães, os pacientes com constipação grave e com constipação leve foram reunidos em um único grupo. Verificou-se hábito intestinal compatível com constipação em $58,8 \%$ das mães das crianças dos grupos com constipação e em $41,2 \%$ das mães das crianças sem constipação (teste do Qui-quadrado $P=0,074$ ). Considerando as 131 duplas de crianças e suas mães biológicas, constipação na criança e na mãe foi observada em 47 (35,9\%) duplas, ausência de constipação na criança e na mãe em 30 (22,9\%), presença de constipação na criança e hábito intestinal normal na mãe em 33 (25,2\%) e hábito intestinal normal na criança e constipação na mãe em 21 $(16,0 \%)$ duplas. De acordo com o teste de McNemar, não se observou discordância estatisticamente significativa $(P=0,134)$ e o coeficiente Kappa igual a +0,16 mos trou concordância muito leve entre a ocorrência de constipação na criança e na mãe.

Quanto à concordância da ocorrência de arco nas 131 crianças e suas respectivas mães, observou-se: arco na criança e na mãe em 20 $(15,3 \%)$ duplas, arco somente na criança em $14(10,7 \%)$, somente na mãe em $31(23,6 \%)$ e em nenhum em $66(50,4 \%)$ duplas. De acordo com o teste de McNemar, observou-se discordância estatisticamente significativa $(P=0,017)$ e o coeficiente Kappa igual a $+0,24$ mostrou concordância leve da presença de arco na criança e em sua mãe.

\section{DISCUSSÃO}

Os dermatóglifos podem ser utilizados para auxiliar no estabelecimento do papel da transmissão genética nas doenças familiares, no entanto, sua utilização clínica deve ser criteriosa, pois nenhum achado é patognomônico e, em geral, os indícios fornecidos pelos dermatóglifos são baseados exclusivamente na maior freqüência estatística de certos padrões numa determinada condição, não permitindo, entretanto, sua aplicação como método diagnóstico individual $^{(1)}$. As vantagens da impressão digital como indicador genético são sua estabilidade durante toda a vida, a rapidez da coleta, baixo custo e seu caráter não-invasivo ${ }^{(1)}$.

O presente estudo foi planejado para verificar se a análise dos padrões de dermatóglifos poderia proporcionar indícios da participação de fatores genéticos e constitucionais na gênese de constipação crônica na infância, a exemplo do observado com outras doenças. Na literatura, existem resultados indicando maior frequiência do padrão arco de dermatóglifos em pacientes com constipação atendidos em serviços especializados em gastroenterologia. Neste estudo, pretendeu-se ampliar as informações sobre o assunto analisando-se, também, pacientes portadores de constipação presumivelmente com menor gravidade. Assim, foram constituídos dois 
grupos de pacientes com constipação: 1. grave, incluídos em ambulatório especializado de gastroenterologia pediátrica, e 2. presumivelmente leve, identificados por rastreamento ativo em serviço de pronto atendimento pediátrico, onde as queixas não se relacionavam ao sistema digestório. Assim, o estudo te ve como base a composição de dois grupos de crianças com constipação, com diferentes g raus de gravidade, que foram comparados com outro g rupo de crianças com hábito intestinal normal. A estratégia para constituir os grupos de constipação resultou em diferentes gravidades, conforme a frequiência das evacuações, a utilização pregressa e atual de laxantes que demonstraram nas crianças incluídas no ambulatório especializado maior gravidade do que nos pacientes incluídos no pronto atendimento, devendo-se ressaltar que no serviço especializado predominou o gênero masculino conforme se re gistra na literatura ${ }^{(13)}$.

A presença de arco foi observada em $25,7 \%$ das crianças com constipação grave, $28,9 \%$ das com constipação leve e em $23,5 \%$ das crianças do grupo-controle, não sendo caracterizada associação estatisticamente significante entre a presença de arco e constipação. Ausência de associação entre constipação e a ocorrência do padrão arco também foi observada em estudo realizado por cirurgiões pediatras $^{(7)}$, envolvendo crianças americanas em que a presença de arcos foi encontrada em 33,3\% de 39 crianças com constipação crônica funcional, em $29,8 \%$ do grupo-controle (constituído por crianças submetidas à herniorrafia) e em $31,5 \%$ dos pacientes com constipação secundária a causas orgânicas como a doença de Hirschsprung e ânus imperfurado. Os resultados desta série e os do estudo americano $^{(7)}$ se contrapõem ao achado em crianças italianas ${ }^{(19)}$, em que arco foi caracterizado em $37,8 \%$ das 45 crianças com constipação (menos de uma evacuação por semana) e em 11,4\% dos 35 controles, sendo a associação estatisticamente signif icativa. Nas crianças com constipação, a ocorrência de arcos foi maior naquelas que apresentavam também dor abdominal. Como pode ser observado, a associação entre arco e constipação no estudo italiano parece estar mais relacionada com a baixa ocorrência de arco no grupo-controle $(11,4 \%)$ do que propriamente por maior proporção de arcos de crianças com constipação $(37,8 \%)$, principalmente, quando estes valores são comparados com os valores observados nos grupos-controle do presente estudo $(23,5 \%)$ e do estudo americano $(29,8 \%)^{(7)}$

Na presente casuística, hábito intestinal compatível com constipação foi encontrado em 51,9\% das 131 mães estudadas. Não se observou maior percentagem de ocorrência de arcos nas mães com constipação $(35,3 \%)$ em relação às mães com hábito intestinal nor mal $(42,9 \%)$. Estes dados diferem de estudo realizado com adultos americanos $^{(8)}$ em que se encontrou associação estatística entre arco e constipação: $27,7 \%$ dos 83 pacientes com constipação e em $11,1 \%$ dos 72 sem constipação. Esse estudo ${ }^{(8)}$ foi realizado em serviço especializado, ou seja, com casos de constipação presumivelmente com maior gravidade, valendo ressaltar, mais uma vez, que a associação parece estar mais relacionada com a menor proporção de arcos no g rupo-controle do que a maior ocorrência de arcos nos portadores de constipação. Este estudo americano ${ }^{(8)}$, também, mostrou associação entre a ocorrência de arco e início de constipação ante dos 10 anos de idade.

Apesar da maior frequiência de constipação nas mães de crianças com constipação, em relação às mães das crianças do grupo-controle, a diferença não atingiu a significância estatística $(P=0,074)$. Por sua vez, o coeficiente Kappa mostrou concordância muito leve entre a ocorrência de constipação nas mães e seus filhos.

Comparando-se as frequiências dos padrões de dermatóglifos obtidos neste estudo tanto nos controles, como nos portadores de constipação, constata-se que a ocor rência desses padrões é semelhante a de outros trabalhos realizados no Brasil( ${ }^{(4,17,21)}$. É interessante mencionar que a freqüência de arcos foi superior nas mães do que nas crianças desta série. Não foi encontrada explicação para este achado. Por sua vez, concordância entre a presença de arco na mãe e no filho foi leve, conforme o resultado do coeficiente Kappa $(+0,24)$.

Assim, vale ressaltar que neste estudo os padrões de dermatóglifos não contribuíram positivamente para o estabelecimento da participação de fatores genéticos no desenvolvimento da constipação, a e xemplo do obser vado previamente na literatura ${ }^{(18)}$

Em conclusão, não se observou associação entre a presença de arco e constipação, tanto nas crianças como em suas mães, sendo muito leve a concordância entre a ocorrência de constipação na criança e em suas mães biológicas.

\section{AGRADECIMENT OS}

Os autores agradecem ao Ser viço de Datiloscopia de Identificação da Polícia Federal de São Paulo (Norma Sueli Guedes Quinhões, Marcelo Cerqueira de Souza, José Abílio de Almeida Magalhães, Carlos Alexandre Fernandes de Paiva, José Manuel Nogueira de Azevedo) e ao Conselho Nacional de Desenvolvimento Científ ico e Tecnológico (CNPq). 
Goshima S, Fagundes-Neto U, Morais MB. Der matoglyphics of children with chronic constipation. Arq Gastroenterol 2004;41(1):37-41 .

ABSTRACT - Background - The dermatoglyphics can be used to study the participation of genetic factors in many diseases. There is controversy concerning the association between the dermatoglyphic patter n of digital arches and constipation. Aim - To compare the der matoglyphic patterns among children with and without chronic constipation in relation to the dermatoglyphic patterns and characteristics of stools of their mothers. Methods - Three groups of children aged from 2 to 12 years and their mothers were studied: 35 patients with severe chronic constipation, 45 children with mild chronic constipation and 51 children without constipation. The fingerprints were taken and evaluated by a datiloscopy technicist and classif ied in arch, radial loop, ulnar loop, whorl and others. Results - Digital arches were found in $25.7 \%$ of severe constipated patients, $28.9 \%$ of mild constipated children and in $23.5 \%$ of controls. There was not a statistical significant association. Constipation w as found in 51,9\% (68/131) of the mothers. Arches were found in 35.3\% of the mother with constipation and in $42.9 \%$ of mothers without constipation. There was a slight association between constipation in children and in theirs mothers (Kappa coefficient $=+0,16$ ). Conclusion - The dermato glyphics were not useful to identify the influence of genetic in constipation. There was not association between the dermatoglyphic pattern of digital arch and constipation both in children and in their mothers. Only a slight concordance was found between constipation in children and in their mothers.

DESCRITORES - Dermatoglyphics. Constipation, genetics. Child.

\section{REFERÊNCIAS BIBLIOGRÁFICAS}

1. Alter M. Dermatoglyphic analysis as a diagnostic tool. Medicine (Baltimore) 1966;46:35-6.

2. Baker SS, Liptak GS, Colletti RB, Croffie JM, Di Lorenzo C, Ector W, Nurko S Constipation in infants and children: evaluation andTreatment. A medical position statement of the North American Society for Pediatric Gastroenterology and Nutrition. J Pediatr Gastroenterol Nutr 1999;29:612-26.

3. Bakwin H, Davidson M. Constipation in twins. Am J Dis Child 1971;121:179-81.

4. Benevides-Filho FRS, Salzano FM. Fingerprints of whites and negroes from southern Brazil. Am J Phys Anthrop 1969;31:59-64

5. Chakravarti A, Blanton S, Kendall BJ, McCallum RW. Cose gregation of familia intestinal pseudoobstruction and presence of digital arches in large multigenerational pedigree. Dig Dis Sci 1996;41:1429-33.

6. David TJ, Ajdukiewicz AB, Read AE. Fingerprint changes in coeliac disease. $\mathrm{Br}$ Med J 1970;4:594-6.

7. Drongowski RA, Coran AG. Dermatoglyphic patterns in children with chronic constipation. Dig Dis Sci 1995;40:1420-2.

8. Gottlieb SH, Schuster MM. Dermatoglyphic (fingerprint) evidence for a congenital syndrome of early onset constipation and abdominal pain. Gastroenterology 1986;91:428-32.

9. Habibullah CM, Mujahidali M, Shivaprakash M, Iqbal MA, Ishaq M. Fingertip and palmar patterns in duodenal ulcer. Hum Hered 1982:32:432-4.

10. Landis JR, Koch GG. The measurement of obser ver ag reement for cate gorical data. Biometrics 1977;33:159-74.

11. Loening-Baucke V. Chronic constipation in children. Gastroenterology 1993; 105:1557-64.

12. Loening-Baucke V. Constipation in early childhood: patient characteristics, treatment, and longterm follow up. Gut 1993;34:1400-4.
13. Maffei HVL, Moreira FL, Kissimoto M, Cha ves SM, El Faro S, Aleixo AM. História clínica e alimentar de crianças atendidas em ambulatório de gastroenterologia pediátrica com constipação intestinal crônica funcional e suas possíveis complicações. J Pediatr (Rio J) 1994;70:280-6.

14. Morais MB, Vitolo MR, Aguirre ANC, Medeiros EHGR, Antonelli EMAL, Fagundes-Neto U. Teor de f ibra alimentar e de outros nutrientes na dieta de crianças com e sem constipação crônica funcional. Arq Gastroenterol 1996;33:93-101.

15. Morais MB, Maffei HVL. Constipação Intestinal. J Pediatr (Rio J) 2000;76:S147S56.

16. Murphy MS, Clayden G. Constipation. In: Walker A, Durie PR, Hamilton JR, Walker-Smith JA, Watkins JB. Pediatric gastrointestinal disease. 2nd ed. St. Louis, Missouri: Mosby; 1996. p.293-321.

17. Penhalber EF, Barco LD, Maestrelli SRP, Otto PA. Dermatogl yphics in a large normal sample of caucasoids from southem Brazil. Braz J Genetics 1994;17:197-214.

18. Preus M, Fraser FC. Dermatoghyphics and syndromes. Am J Dis Child 1972;124:933-43.

19. Staiano A, Andreotti MR, Perrotta V, Strisciuglio P. Prevalence of digital arches in children with abdominal pain and constipation. J Pediatr 1990;117:435-6.

20. Swartz MH, Herman LMV, Teichholz LE. Dermatoglyphic patterns in patients with mitral valve prolapse: a clue to pathogenesis. Am J Cardiol 1976;38:588-92.

21. Tahan S, Medeiros EHGR, Wehba J. Padrões der matoglíf icos na doença celíaca. Arq Gastroenterol 1997;34:196-203.

22. Talle y NJ, Weaver AL, Zinsmeister AR, Melton III LJ. Onset and disappearance of gastrointestinal symptoms and functional gastrointestinal disorders. Am J Epidemiol 1992;136:165-77.

23. Walker NF. Inkless methods of finger, palm and sole printing. J Pediatr 1957;50:27-9.

Recebido em 23/12/2002. Aprovado em 13/8/2003. 